\title{
Considering rich spatiotemporal relationships in Cultural Heritage information management
}

\author{
Pierre Hallot ${ }^{1}$ \\ ${ }^{1}$ Liège Université, Faculty of Architecture, DIVA-LNA Belgium \\ p.hallot@ulg.ac.be
}

\begin{abstract}
This paper describes the use of rich spatiotemporal relationships in cultural heritage information modelling in order to increase the information organization and extraction. The idea is to tailor the spatiotemporal state of identity model, i.e. a model based on spatiotemporal identity that takes into account relationships between non-existing and non-presents objects, to the management cultural heritage information. In doing so, we propose an enhancement of the knowledge representation for cultural heritage.
\end{abstract}

Keywords: spatiotemporal relationships; cultural heritage information; life and motion configuration; existence; presence; identity.

\section{Introduction}

Cultural Heritage information management is impacted by the evolution of the acquisition techniques, mainly by the arising of the laser scanner technology [1]. The digital transformation conduces to an increase of the acquisition performance, the completeness of acquired information and the evolution of the represented information acquired from such technologies. Managing digital heritage data requires systems that are able to integrate the complexity of cultural heritage information, i.e. managing the several levels of temporality involved in the life-cycle of a represented element [2]. Geographical information science is, among others, attached to the definition of theoretical concepts that are implemented in information management systems. Following the same approach, the theoretical definition on relationships leads to a refinement of concepts closely tied to space and time that the first step for semantic enrichment of cultural heritage information.

For years now, the development of spatiotemporal relationships models was predominantly dedicated to the management of geospatial information [3]. Although, cultural heritage information owns a spatial component, the main difference resides in the temporal dimensions that is a fundamental descriptor for such kind of information. The description of the evolution of an entity is based on the study of the succession of spatial and temporal states that symbolize the life cycle of the object. Moreover, the 
accessibility to the information is rather limited or incomplete. Accessing to a cultural heritage information is performed through one of the following ways:

- identity: the identity of represented cultural heritage element is known. There is no ambiguity on its identification. The information can therefore be structured around object's identity. E.g. the pyramids of Giza in Egypt.

- physical realization: the cultural heritage element is only known by a physical structure visible in the real world. The spatiality can be represented but is not necessarily linked to a defined or unique identity. The link between the physical observed elements and the object identity is not sure. E.g. an unidentified ruin.

- spatiality: the spatiality of the object is known without manifestation in the real world. Information related to the geometry of the element are known but there is no physical manifestation of the element in the real world. The spatial position can be known or not. E.g. the plan of La Bastille in France.

Each of the information accessibility way faces to an uncertainty. Indeed, information related to cultural heritage objects results of sources interpretation performed by a operator who has a vision of the world that relying on its experience. Several interpretations or evolution scenario are frequent in cultural heritage information management. Developed information systems have to consider this ambiguity to fully encompass the cultural heritage information.

In a previous research [4, 5], we proposed a spatiotemporal states (STS) model that defines spatial and temporal relationships between (geographical) objects in considering their whole range of possible existence states. The commonly used spatial relationships only deals with two objects that exists at the time of the analysis. Starting from a description of the possible states of an object in regards of its identity, spatiality and presence, we defined a set of possible states of existence and presence for a geographical object. This classification is applied to several object to deduce extended spatiotemporal relationships. For example, the STS model proposes anachronistic relationships between an object that exist with another object that does not exist physically anymore.

In this paper, we throw the bases for an application of the STS model to cultural heritage information management. Our research hypothesis is that considering refined spatiotemporal relationships between heritage objects ensure a better description of the complex cultural heritage information. We postulate that spatiotemporal relationships based on the STS model will carry more semantic for the relationships description.

The remaining of the paper is structured as follows. First, we remind the concept of spatiotemporal states of existence and presence. Then we expose the main issues in cultural heritage information management. The second part shows the adaptation of the STS model to deals with cultural heritage information in considering object identity as a starting point. This section symbolizes the life cycle of cultural heritage object in the regard of the evolution of its identity. Finally, we introduce the concept of relationship observer that refines the proposed relationships by considering the temporal point of view of a relationship between objects. Then we conclude. 


\section{Spatiotemporal states of identity}

The identity of an object is the property intrinsic to each object which allows it to be differentiated from all others [6]. The complete definition of a geographical object involves the definition of its identity and spatiality, which can vary over time. Research focusing on the identity of geographical objects [7] focuses on the concept of a geographical object and its modeling as a unique object. Campos [8] proposes a set of relationships and their associated semantics describing the representation of geographical objects in a virtual environment. Although this approach can be linked to cultural heritage information management, the model does not take into account the possibility to establish relationships between an object present at the moment of the analysis as against an object that existed in the past or an event existing in the future. Spatiotemporal relationships such as that proposed by Claramunt [9, 10] define a set of spatiotemporal relationships that integrate topological reasoning with the temporal logic. These models describe, by means of qualitative operators, the relationships that exist between moving objects which are only existing and visible during the analysis.

The spatiotemporal states of identity model (STS-I) has been initially developed to fully encompass the complexity of geographical information $[4,5]$. Indeed, there exist a lot of situation in the geographical domain where the spatiality of a geographical object cannot be acquired, at least for some period of time. In order to propose a continuous analysis of the geographical object over time, the STS-i model has been developed focusing on the objects identity. Several spatiotemporal states have been outlined in regards of spatial, temporal and identity vision. The Figure 1 shows the organization and the transition between spatiotemporal states. Since it is not possible to model every objects that exists or that have been existing over time, represented objects are limited to the ones that are pointed by a relationship. The existence of an object begins as soon as a semantic or spatial relationship is established for this object and that it ceases to exist when there are no more relationships with it. Once the object identity is proposed, the object switch from the "non-existent" state to the "existent" state. This state does not define a physical realization or a spatial design of the object. The materialization operation provides an extension in the physical world for the object. At that moment, the object owns an identity, a spatiality and a physical representation. We qualify this state of "present". When the physical realization of the object is destroyed or if the spatiality is planned but not yet materialized, the object is in a non-present state. This state gathers either the objects that are destroyed with a souvenir of their spatiality or the objects for which spatiality is planned but not yet visible in the physical world. These different cases do not entirely correspond to a lack of information or vague information because the identity of the object very often remains known. Loss of information about the temporary spatial extension does not destroy the existence of the geographical object. 


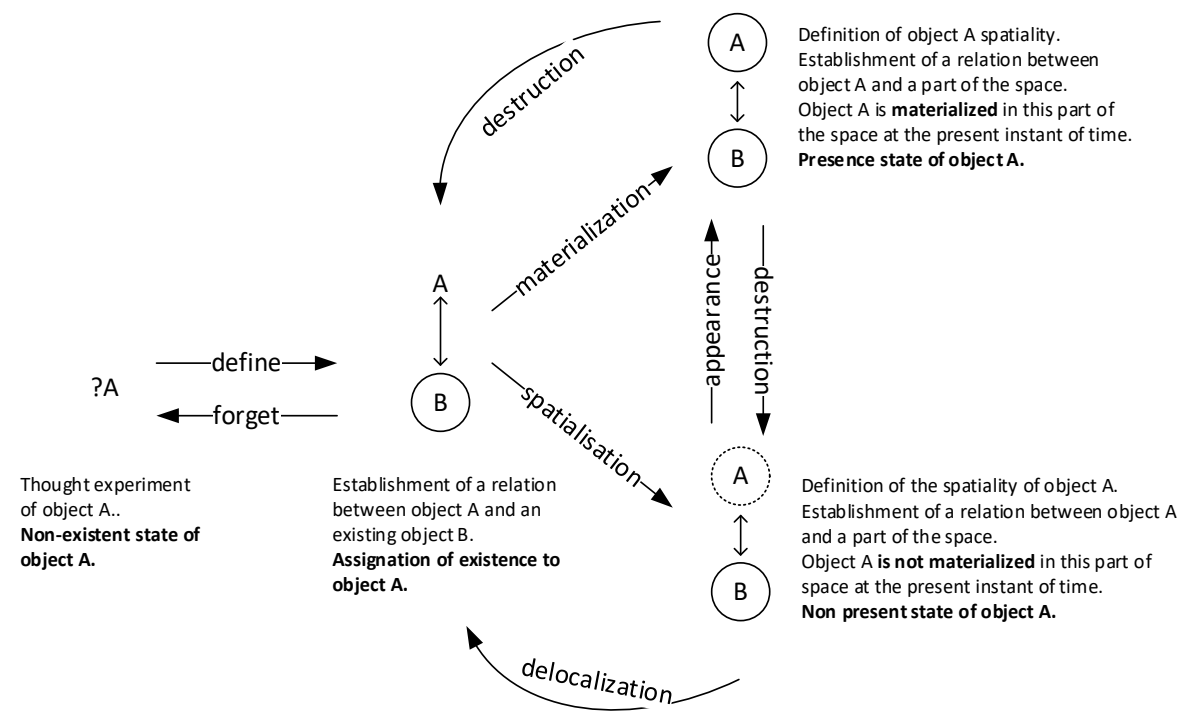

Fig. 1. Conceptual schematization of the different spatiotemporal states of an object A as defined in [4]. The arrows represent the transition events between these different states. The destruction corresponds to an omission of the location of the object; the delocalization marks the loss of the realization in the physical space without omission of its position. The destruction symbolize the loss of spatial extension, which is either with loss of spatiality or not.

When combined together in regards of two objects, the succession of spatiotemporal states of identity of two objects defined the Life and Motion Configuration (LMC). The LMC is an iconic language that symbolize the evolution of spatiotemporal relationships between two objects considering their relationship at each temporal succession.

\section{Application of STS-I to Cultural Heritage Objects}

The application of STS-I model to cultural heritage objects requires some adaptations to fit to the complexity of cultural heritage concepts as described in the introduction. When dealing with geographical information, the succession of spatiotemporal states of identity is largely linear, starting from non-existence, existence, non-presence and presence. The destruction scheme follows the inverse succession. However, building archeology or conservation/restauration research either leads to the discovery of unidentified physical fragments or faces to sources asserting the identity and/or the spatiality of objects without physical realization. The lifecycle of cultural heritage buildings is complex due to the multiple transformation, affectation change or the ageing due to time. The representation of cultural heritage information with the prism of spatiotemporal states of identify gives a formal classification of every states of the 
historical elements. Figure 2 shows the evolution of an object for which the historical knowledge is complete, this means that each of the transformation, or planned transformation is documented and can be established by undoubtable sources. The first step in time represents the identity of the object. At that time, there is not yet a definition of any spatial information of the element. The second step is obtained after a stage of architectural conception, which is attached to the pacification, and the definition of the spatial extension of each part of the building. The construction phase leads to the first state of the edifice. Most of spatial and temporal reasoning starts at that step in the object definition. Long-term evolution representation implies to consider multiple changes that occurs to modelled objects. In cultural heritage information modelling, each historical sources provides information on physical or thematic changes that affects modelled object. When planning a transformation, there is a concurrent existence between the physical realization and the spatial definition of the next state. Once the transformation is done, the new physical realization appears and the object switch from state 1 to state 2 . At the end of the lifecycle, the physical realization of the edifice disappears; the spatiality continues to exist for a while. The heritage building is qualified of non-present. At the ends, when no information on the former spatiality of the edifice remains, e.g. the plans are lost, only the identity of the elements continues to exist.

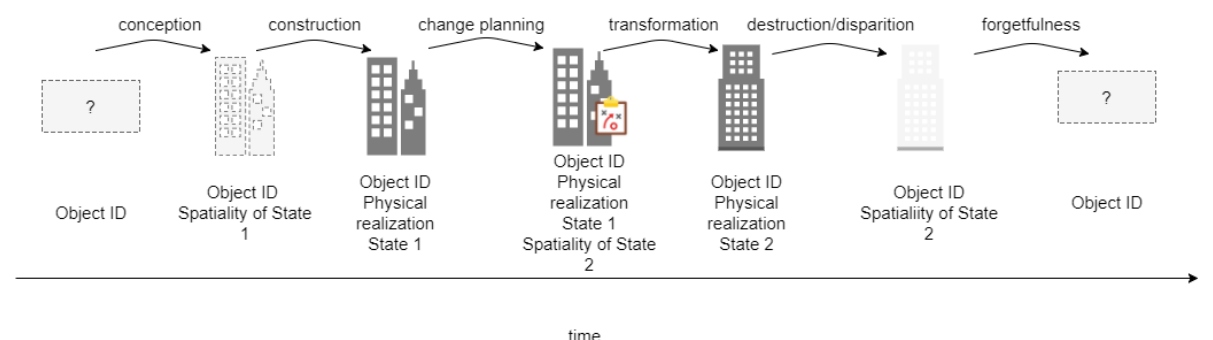

Fig. 2. Conceptual schematization of successive spatiotemporal states of a heritage building. The figure shows, for each state, the knowledge on the identity, the spatiality and the physical realization of the object. The arrows represent the transition events between these different states.

The organization of heritage information around the spatiotemporal states allows sketching what information was available at a fixed time in history. Moreover, it refines the traditional spatiotemporal representation in allowing the concurrence of what is visible in the physical world with what is planned for the future. In the same way, each previous states can be stored and represented independently of their temporality, i.e. in proposing a view of all states of the heritage edifice evolution.

When considering incomplete knowledge on the object evolution, the information acquisition is done through sources that have to be interpreted [11]. The representation of incomplete knowledge and fussy knowledge remain an open issue [12, 13]. The spatiotemporal states are then subjective results that can leads to several concurrent interpretation scenarios. By recording every planned transformation, even if they are not all implemented, we allow to store and organize comprehensive knowledge on 
cultural heritage elements. Planned others spatiality's is of a high importance for researchers to fully understand the lifecycle and the history of the heritage buildings and to allow a classification to every sources related to build heritage. There is actually no degrees of confidence related to the interpretation of the spatio-temporal states. We plan to consider this perspective in a future research.

\section{Anachronistic relationships for cultural heritage objects}

The transformation or the restauration of the built heritage has to take into account the history of the building and surrounding environment it works on. More specifically, if the built heritage has been transformed or partially destroyed, the vision of the former space has to be considered during the conception of the architectural project. For example, the restoration of a heritage building which is, among others, composed of an empty space at the present time has to integrate the former role and the former geometry of what that space was. The proposed project will have to consider the past state, usually by proposing a symbolization of that former spatiotemporal state. In doing so, we draw a spatiotemporal relationship between built heritage at different period of time. This is what we define as an anachronistic relationship, i.e. the spatiotemporal relationships between objects states that were not existing in during a concurrent period of time.

Going deeper in the refinement, we assume cases where the anachronistic relationships points on the object spatiality, the object physical extension or only to the object identity. It is important to propose information management systems that allows to store and describe such kind of relationship because of their fundamental aspects to understand architectural planning. Figure 3 represents two kinds of anachronistic spatiotemporal relationships. The blue area shows an architectural project which is still in conception phase.

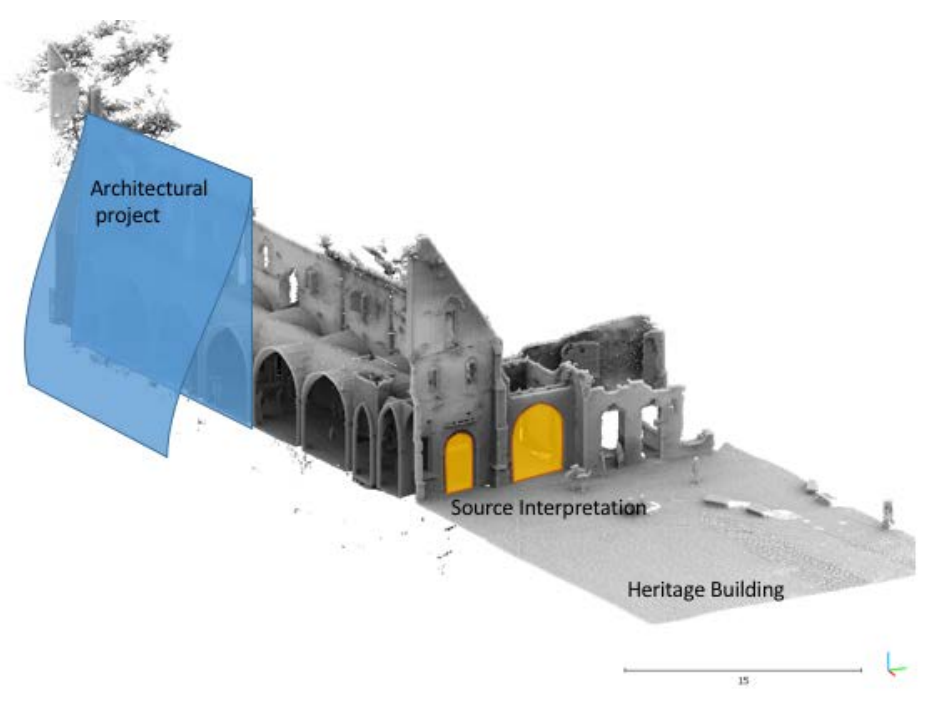


Fig. 3. Anachronistic representation of several states on a heritage building representation. The architectural project is a planned spatiality of the building evolution. The source interpretation is an anachronistic representation of past-existing doors of the building added a contemporary representation of its actual shape.

This means that the identity of the project is known, the spatiality is being designed but the project does not have an extension in the physical world. This object is in relation with a heritage building (represented by a laser scanner acquisition in the figure). The second part of the figure 3 shows some elements (the doors) that do not have a physical extension but only a spatiality at the present time. Their representation gives an interpretation of the relationships of their actual spatiotemporal state (non-present) with the building (present). Such kind of representations helps at understanding the relationship that was existing between the two elements at the time when they were both present (i.e. with a spatial extension).

The previous examples show that a spatiotemporal relationship are used to conceptualize the relations that exists at every time between buildings elements. This helps first at organizing the historical sources or their interpretation, and secondly to understand and plan future architectural projects which has to consider the historicity of the context. These two approach lead to think at the targeted goals of establishing such spatiotemporal relations. The role of the operator is significant in order to define at what time the relationship is observed and consequently what will be the use of the relationships. Let assume that two objects A and B share a spatiotemporal relationship, which remain stable over time (the objects does not change of state). The relation can be observed in three ways:

- $\quad$ as it was in the past:

This view of the relation is used each time when an historical study is performed. We try to retrieve what was the relationships in the past.

- as it is now:

This view of the relation corresponds to an actual observation of the spatial organization of past elements. For example, the relation is used to explain the actual spatial organization of a former medieval city, which is influenced by the link that exist between defense walls and the surrounding houses.

- $\quad$ as it will be for the future:

This view of the relation is used in planning when transforming one of the object participating to the relation. It will affect the spatiotemporal relation. 


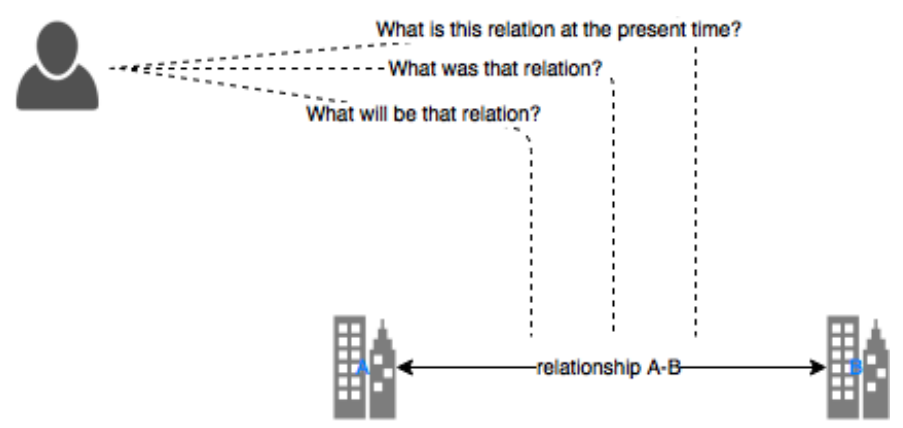

Fig. 4. Temporal interpretation of spatiotemporal relationships depending on the observer. The relationships between objects can be studied as it was in past (at the time objects were both present), as it is actually (what does the relationships implies in the present), as it will be in the future (how the relationship will be considered in the future).

Anew, the conceptual refinement of a relationship by considering the temporality of the observation helps at organizing the cultural heritage information and leads to a better sources interpretation. This helps at understand the spatial choices that are continuously taken in architectural design.

\section{Conclusion}

The cultural heritage information management has to consider multiple historical sources. Their interpretation conduces to different scenario that can sometimes be contradictory. To fully understand the lifecycle of built heritage, relationships between actual and former states of elements has to be drawn. In this paper, we proposed to adapt a spatiotemporal relationships model based on object identity to test if it could fit to cultural heritage information modelling. This first conceptual step aims at describe the general concept where the STS-I model can be applied and the uses that can be done in this context. The first results are promising and should be now defined formally before being implemented and tested. This next research step will be considered soon with real cases examples on the "Hotel Rigo" in Liège, Belgium. This case is highly interesting since is should be destroyed in favor of an urban development.

\section{Bibliography}

1. Yastikli, N.: Documentation of cultural heritage using digital photogrammetry and laser scanning. J. Cult. Herit. (2007).

2. Meyer, É., Grussenmeyer, P., Perrin, J., Durand, A.: A web information system for the management and the dissemination of Cultural Heritage data. Cult. Herit. (2007).

3. Stock, O.: Spatial and temporal reasoning. Kluwer Academic Publishers, 
Dordrecht ; Boston (1997).

4. Hallot, P., Billen, R.: Enhancing Spatio-Temporal Identity: States of Existence and Presence, (2016).

5. Hallot, P., Stewart, K., Billen, R.: Les états spatiotemporels d'existence et de présence. Int. J. Geomatics Spat. Anal. 25, 173-196 (2015).

6. Khoshafian, S.N., Copeland, G.P.: Object identity. SIGPLAN Not. 21, 406416 (1986).

7. Hornsby, K., Egenhofer, M.J.: Identity-based change: a foundation for spatiotemporal knowledge representation. Int. J. Geogr. Inf. Sci. 14, 207-224 (2000).

8. Campos, J., Hornsby, K., Egenhofer, M.: A temporal model of virtual reality objects and their semantics. J. Vis. Lang. Comput. 14, 469-492 (2003).

9. Claramunt, C., Jiang, B.: An integrated representation of spatial and temporal relationships between evolving regions. J. Geogr. Syst. 3, 411-428 (2001).

10. Claramunt, C., Theriault, M.: Toward semantics for modelling spatio-temporal processes within GIS. Adv. GIs Res. I. 27-43 (1996).

11. Van Ruymbeke, M., Carré, C., Delfosse, V., Pfeiffer, M., Billen, R.: Towards an Archaeological Information System: Improving the Core Data Model. In: Giligny, F., Djindjian, F., Costa, L., Moscati, P., and Robert, S. (eds.) CAA 2014 21st century Archaeology : Concepts methods and tools : Proceedings of the 42nd Annual Conference on Computer Applications and Quantitative Methods in Archaeology. pp. 245-253. Archaeopress, Paris, France (2015).

12. de Runz, C., Desjardin, E.: Imperfect Spatiotemporal Information Analysis in a GIS: Application to Arch\{æ\}ological Information Completion Hypothesis. In: Jeansoulin, R., Papini, O., Prade, H., and Schockaert, S. (eds.) Methods for Handling Imperfect Spatial Information. pp. 341-356. Springer Berlin Heidelberg, Berlin, Heidelberg (2010).

13. Belussi, A., Migliorini, S.: A spatio-temporal framework for managing archeological data. Ann. Math. Artif. Intell. 80, 175-218 (2017). 